\title{
Model-Driven Generation of Collaborative Virtual Environments for Cultural Heritage
}

\author{
Alberto Bucciero ${ }^{1}$ and Luca Mainetti ${ }^{2}$ \\ ${ }^{1}$ National Council of Researches, Institute of Archaeological and Monumental Heritage, \\ Rome, Italy \\ alberto.bucciero@cnr.it \\ ${ }^{2}$ University of Salento, Department of Innovation Engineering, Lecce, Italy \\ luca.mainetti@unisalento.it
}

\begin{abstract}
Collaborative Virtual Environments are experiencing a large interest in cultural heritage field mostly due to the strong opportunity given by novel augmented reality applications. There are already several examples of collaborative augmented visit to museums or historical sites. Anyway the traditional and static approach to computer graphics is very limiting because it often requires the development of a Virtual Environment for every new application.

In this paper, we propose a technique for model-driven generation of mixed reality virtual environments, where every modification in contents, visit path and in interactions with the physical surrounding environment don't require a great re-coding effort, enabling fast deployment of collaborative virtual environments for cultural explorations only providing new contents and a small set of parameters.
\end{abstract}

Keywords: collaborative virtual environment, conceptual design, conceptual map, mixed reality, multi-user virtual environment, 3D virtual world.

\section{Introduction}

Collaborative Virtual Environments (CVE) are becoming more and more important in many areas; their use goes from military training and simulation, to sharing of scientific data between scientists or decision-makers (CSCW), from support for innovative teaching paradigms, to support for collaborative e-learning (CSCL). Also, they are becoming one of the most promising multimedia technologies used to preserve and explore cultural goods [1].

One of the traditionally and oldest application field of CVE is virtual reconstruction of real or imaginary places for cultural heritage, for example in [2] a multi-user 3D world supports users in virtually visiting the Qumran museum to overcome the difficulties due to the geographic region where it is located.

Another interesting example is in [3] where users interact in real time from remote locations with 3D archaeological models through a shared virtual environment using tele-immersive technology. 
Starting from a definition, a CVE (Collaborative Virtual Environment) is a virtual 3D place representation, in which users, being represented with graphical embodiments called avatars, share the same experience, working and interacting with the same set of virtual objects. In this case users see the same representation of shared workspace (i.e. a 3D world) and the effects on the users' or objects' actions are also the same for all. This paradigm is known as WYSIWIS ("What you see is what I see").

The availability of mobile location aware technologies on consumer devices encourages researchers to consider how to join the classic desktop CVE paradigm with a newer one based on mobile devices, in a mixed reality oriented fashion. Then the paradigm is not anymore "WYSIWIS", like a classic Collaborative Virtual Environment, but WYSINWIS ("What you see is NOT what I see"): users see different representation of the same workspace (both environment and objects).

Such systems are called CMVRs (Collaborative Mobile Virtual Reality systems) in which avatars in the virtual world and mobile players in the real world share the same experience, aiming to a common goal even if provided with different devices, with different capabilities and then with different views of the same workspace.

Mixed and augmented system involving 3D visualizations and mobile units are extensively used in virtual archeological reconstructions (e.g. ARCHEOGUIDE [4] and MUSE [5]) and collaborative exploration of museum exhibitions (see e.g. [6]). The equipment used typically involves HMD (head-mounted display [6]), wearable laptops and tablet PCs $[4,5,6]$ and PDAs [4, 6]. The positioning of the user is determined by direct user input, recognition of images of corresponding landmarks taken by wearable camera [4] or GPS [6].

The design of such application is however very difficult, because on the one hand the interactions between users and workspace in the 3D environment are substantially different from those that could be supported by mobile devices, on the other hand it is very important being able to design environment, interactions and contents of both $3 \mathrm{D}$ and mobile 2D without loosing sight of the main goal of the collaborative session (oriented to learning, gaming, virtual visit, etc.).

Even if CVEs are more and more widespread and technologically advanced, they are often still developed starting from the environmental container, tightly coupled with them. The development of the 3D environment, that represents the stage where the action and interaction takes place, often lead designers to define action/interaction rules on the basis of the environment facilities offered by it, and not the exact contrary as it should be. In CMVRs this cannot happen because the shared workspace is not homogeneous, typically it is partially $3 \mathrm{D}$ (on traditional workstation) and partially 2D (on mobile devices). Then it is needed a design approach that let the designers free themselves from technicalites and that let them focus the attention over the goals that the collaborative session is aiming.

In this paper we present a technique for model-driven generation of mixed reality virtual environments, where every modification in contents, visit path and in interactions with the physical surrounding environment don't require a great re-coding effort. The paper is structured as follows: section 2 reports essential related work. Section 3 provides readers with an introduction of our model-driven approach to CVEs, stating the design problem, the proposed conceptual solution, and a real case study in 
the cultural heritage domain. Finally, in section 4 the conclusions summarize our key messages and sketch future research directions.

\section{Related Work}

In the international scientific panorama there are many examples of research works related to the CVE and, in particular, of those oriented to the cultural heritage. We present here an overview of the approaches to the design of the virtual environment and we focus on the conceptual layer of design: all these approaches demonstrate that even if CVEs are widespread, the problem of their conceptual design is far to be solved.

The traditional virtual environment design techniques are based on real world concepts and the usual approach is to design just the real situation, translating physical concepts in a $3 \mathrm{D}$ virtual world.

Collaborative Virtual Real Environment (CVRE) [7] was developed by the Universidad du Chile and it models the virtual environment using the concepts of the real world such as rooms, auditorium and so on.

Another approach to the conceptual design is Process Modeling Language (PML) [8]. It is derived by UML and has two levels: High Level UML-Based Diagram (in which it uses P-activity to design the activities and P-class to design objects) and Low Level Process Language, an Object-Oriented language that provides a representation of the classes defined in the P-class using methods defined in the P-activity. The output of the Low Level Process Language may be used in the graphical engine to create the $3 \mathrm{D}$ environment.

NiMMiT (Notation for Modeling Multimodal Interaction Techniques) [9] is another technique to design the computer human interaction. It is based on a state chart graphical notation, oriented to virtual environments.

Considering the semantic gap between designers and developers of CVE, a new technique was thought: the InTML (Interaction Techniques Markup Language) [10]. It is a domain specific language made to define several aspects of a virtual environment (device, objects, interactions). InTML uses a dataflow architecture where objects and device are part of the flow, and are linked each other.

Whereas these approaches provide technical designers (mainly engineers) with support for modeling the graphical or behavioral aspects of $3 \mathrm{D}$ virtual worlds, they lack in characterizing the semantics of the user interaction and collaboration in CVEs. To cope this lack, we propose to extend the semantic perspective to establish a common ground between designers of collaborative experiences and CVEs engineers, and to provide guidance in particular for cultural heritage domain experts.

\section{$3 \quad$ Mixed CVE Conceptual Design}

\subsection{The Design Problems}

The problem of the conceptual modeling of virtual environments has been traditionally faced, as described in the state of the art, using an approach that forces the designer 
to learn technical formalisms, distracting him from the aspects more related to the specific conceptual modeling. Therefore, domain experts (archaeologists, historians, sociologists, etc.) are forced to acquire some technical skills just to be able to give requirements to developers and $3 \mathrm{D}$ graphic designers.

To enable directly domain experts to model CVEs, the number of elements the designer must keep under control must be reduced, focusing on those that are related to the goal of the collaboration and social interaction. In other words, it is needed to add the conceptual perspective to CVE design. The approach must be easy and intuitive, simply understandable also for the virtual environment designers and from which a formal representation can be derived, to can be used to generate CVEs. The best strategy to define a conceptual level is, in our opinion, to think to a metaphor (intended as "a figure of speech and or phrase that one word as being or equal to a second object in some way"). This metaphor should be able to represent the conceptual primitives for the CVE modeling and should be abstract enough to be used in several contexts.

Moreover defining adequate general concepts, abstract from technology-specific details, we can devise a representation of a CVE independently from the specific technical platform used, and we can think to create a sort of "compiler" that would translate the unique abstract representation to a concrete implementation ready to be deployed on specific classes of devices (workstations, smartphones, tablets, etc.).

In order to reach this goal, we carried out an experiment capturing the concepts that underlie the theatre domain and representing them with a concept map.

\subsection{Proposed Solution: The Map of Concepts}

Evolving our virtual world engine WebTalk [11] and its mobile version [12] during past six years - in collaboration with the HOC-Lab of Politecnico di Milano under the L@E, S@L, L@SS, and L4A (Learning for All) projects -, we faced a number of issues trying to make highly configurable our technology. To rise in generality, we have been driven by the need to capture all the relevant abstractions embodied in CVEs for several domains (education, cultural heritage, and sport ethic). These would have been a clear guideline to design the software architecture. The abstraction process has been the result of several iterations that involved on the one hand technical experts and on the other hand conceptual modeling and domain experts in analyzing CVEs and 3D virtual worlds.

The result is presented in the "concept map" presented in Fig. 1. The concept map is a diagram showing the relationships among CVE's recurring concepts. In Fig. 1 concepts are represented as nodes, while relationships are symbolized as labeled arrows. Labels express the informal semantics of relationships. As readers can see, we identified two different sets of concepts: static concepts (white nodes) and dynamic concepts (dark nodes). They capture in a separate layer two aspects of CVEs: (1) the world appearance and its spatial organization, (2) the interaction among users and between user and the word. Analyzing Fig. 1, readers can observe the presence of some fundamental concepts in CMVR design, that in the following are described as theatre concepts: 
- $\quad$ Theatre: a building (i.e. a finite space) where a performance takes place.

- Stage: a large platform on which actors can stand and can be seen by an audience.

- Scenography: a description of a stage that illustrates the environment organization in term of space, set, costume, sound, lighting, etc.

- Script: the written text of a stage play.

- Act: a segment of a performance of a theatrical work; it is an organizational part.

- Scene: a division of an act presenting continuous action in one place. It is the shortest autonomous (semantic) unit in the script.

- Actor: a theatrical performer, i.e. a person who interprets a dramatic character or personality.

- Director: a person who oversees and orchestrates the theatre production usually with responsibility for action, lighting, music, and rehearsals.

- Scene director: a person who is responsible for the stage set up. He/she can modify the original configuration.

- Prompter: a person who assists (one acting or reciting) by suggesting or saying the next words of something forgotten or imperfectly learned. He/she gives the actors the opening words of each phrase a few seconds early.

- Stage whisper: a loud whisper by an actor that is audible to the spectators but is supposed for dramatic effect not to be heard by one or more of the actors.

Actors have a central role, because they are the protagonists of the virtual environment many other concepts are related to this one.

The Curtain is the entry point of the Stage the virtual environment is composed of. When Actors access a Curtain, they must be identified; then, depending on their Roles, the environment configures itself consequently.

Moreover, a relationship between Curtain and Script has been considered: the Curtain could change the Script that, in turn, is related to the Actors.

It is important to note the presence of a Director for the specific virtual space. A Director is a particular Actor who acts as a guide or a tutor for other users during the virtual sessions. In other words, a Director is a staff member that make sure that the action is moving along predefined lines, taking care of the collaborative mixed experience goal.

\subsection{Case Study: The SIBECS Project}

To test the approach we used the SIBECS project scenario. SIBECS was a project founded between 2007 and 2009 by Apulia region that aimed carrying out, experimenting and promoting innovative fruition services to access (and at the same time to preserve) the cultural heritage. One of its results was to develop a mixed reality walkthrough between the modern city of Lecce and its ancient core, Lupiae, discovering "Points Of Interest" (POIs) near their visit path. In this collaborative experience, standard users log on a tridimensional environment in which they can access the contents in collaboration with other connected users, both standard and mobile ones. 


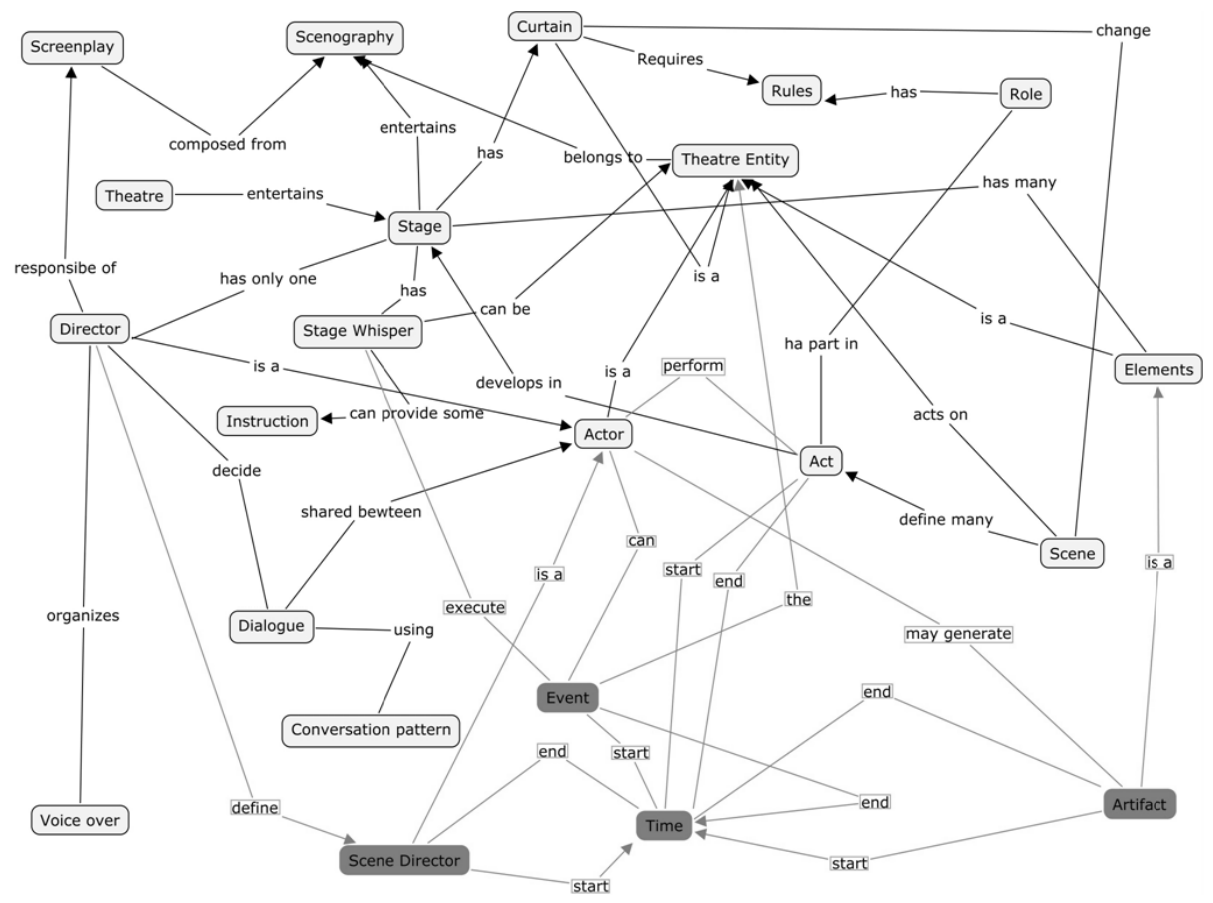

Fig. 1. Map of CVE concepts

Users, showed in the 3D environment (Fig. 2) as avatars, can discover new details about a POI using this the 3D channel. On the other hand, the users equipped with a smartphone can browse the tours using a geo-referred map (Fig. 3). When a POI is near the user, its detailed description will appear automatically on the display. Standard and mobile users share a conceptual workspace: standard users walk in a 3D reconstruction of the city while mobile users are really there in the same time, and can interact to each other chatting, raising events (for example the first who arrives in a place force a video to be played on all other devices), discovering POIs, etc.

When we first worked at the SIBECS project (2007-2009), the deployment of a collaborative session was still hand coded. After having developed our generative approach (starting from 2010), an experiment has been carried out re-writing (remodeling and generating) the SIBECS cultural application. Every concept has been expressed exploiting the theatre metaphor and, thank to an ad hoc editor (see Fig. 4), the CVE model of the SIBECS environment has been translated (mapped) in the configuration script that has been provided as input to the WebTalk engine.

\subsection{Architecture of the CVE Configuration Editor}

In order to simplify the configuration phase, the CVE editor has been designed as wizard whose steps drive the users in the definition of each concept of the conceptual 


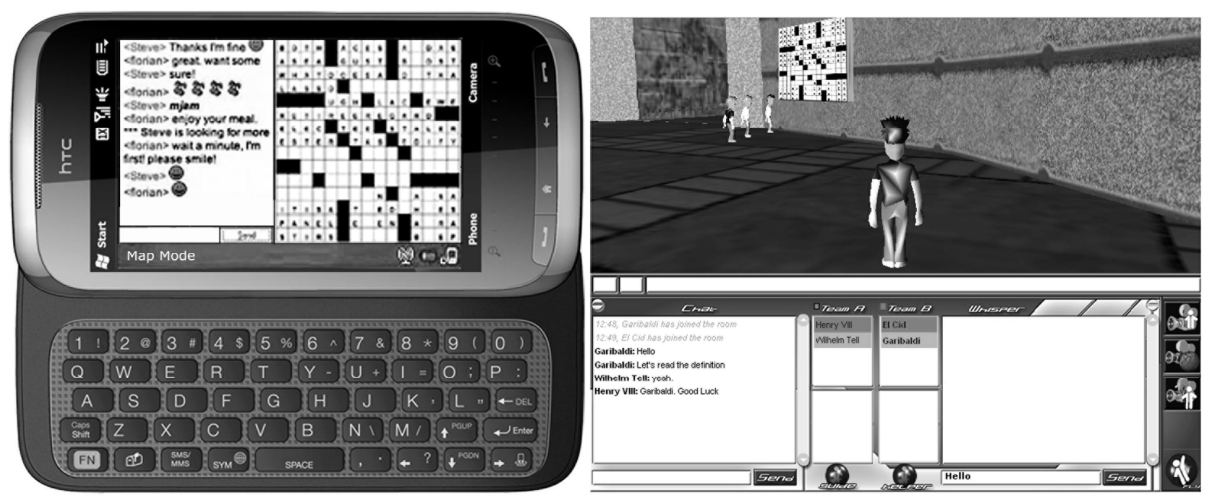

Fig. 2. Collaborative 3D standard and mobile application

map. The interface is structured like the open page of a moleskine, a note pad used to record thoughts and sketches. The left side is occupied by a label that contains suggestions to the user on what to do in the front right are the controls required by the design.

For example, the screenplay page (Fig. 4) has within four controls:

1. Text Box n.1, where you can enter the name of the script

2. Text Box n.2, where you can enter the plot of the opera

3. Numerical textbox with arrows to automatically change the value entered, to set the number of acts

4. Validation control that displays in real time the errors and diagnostic messages for their correction

The wizard is developed in Microsoft Silverlight and its architecture can be divided in the following modules:

\section{Model}

2. Wizard logic

3. Views

4. XMI serializer

5. Webservice access

The software modules composing the system architecture can be logically arranged into three distinct functional layers:

\section{Presentation}

2. Business logic

3. Communication

The Presentation layer contains all the classes that interact with the end user, their goal is to show the data obtained from the model and to process user input. These 


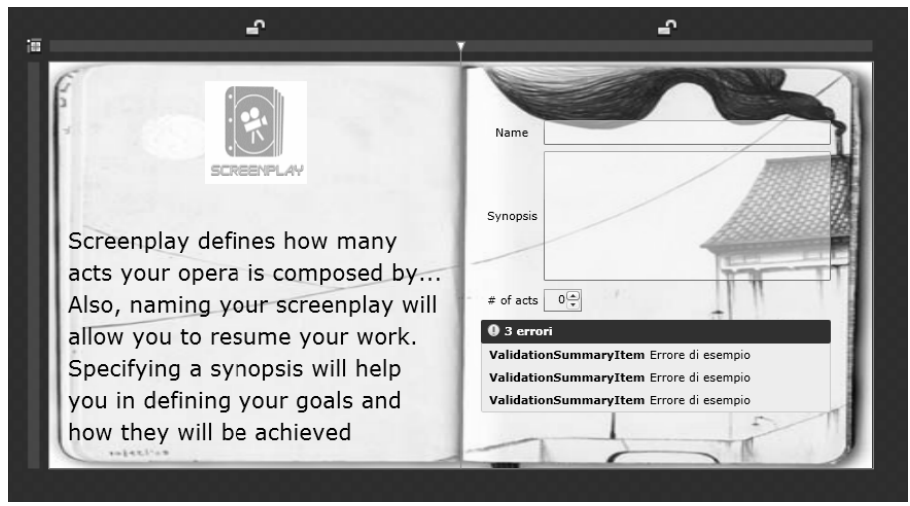

Fig. 3. A screenshot of the editor of CVEs based up on the theatre metaphor - screenplay interface

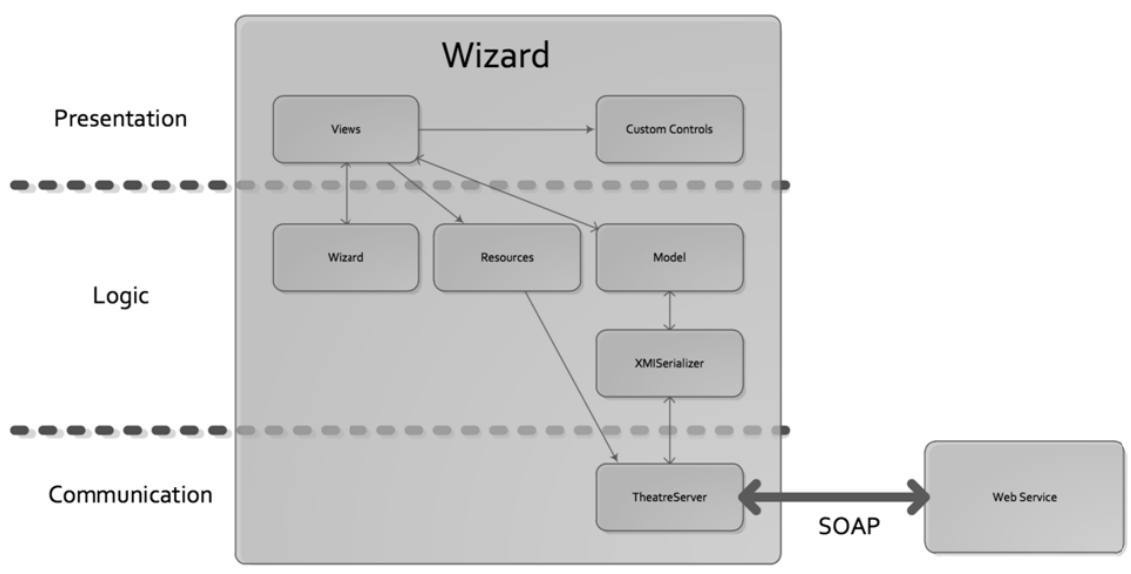

Fig. 4. Wizard software architecture in the large

classes are included in the Views module, and can use extensions to user controls in Silverlight Controls defined in the module.

The Business Logic layer contains all the classes that manage instances of conceptual map and the inner workings of the software. Other significant modules are:

1. Resources, which manages the resources associated with the elements of theater (stage, size, ...).

2. Wizard, which provides live validation of the concepts instantiated and gives feedbacks to the user.

The communication layer is responsible for access and communication with the server, it is composed of modules: 
1. Serializer, which provides the serialization of models in XMI to allow interchange with the WebService.

2. TheatreServer, who takes care of communication with the WebService the purpose of receiving the set of resources and exchange instances XMI and other messages.

\section{Conclusion}

The growth of CVEs for cultural heritage (but also for other domains as education, entertainment, marketing, and collaborative work) calls for new conceptual tools that enable domain experts and engineers to model and keep under control the design complexity unleashed by innovative $3 \mathrm{D}$ virtual worlds, and carefully consider the impact of the design decisions on the optimal flow of the user experience during online sessions. To meet this challenge, we devised a set of concepts organized in a conceptual map that we used instantiate our mixed collaboration engines: WebTalk and Mobile Webtalk. The case study carried out in the cultural heritage domain demonstrated the promising advantages of our approach: expressiveness to capture collaborative features at a high level of abstraction, semi-formality to facilitate the establishment of a common ground between designers of educational experiences and CVEs engineers, and guidance to enable non-experts to cope with all the relevant aspects of a $3 \mathrm{D}$ virtual world.

In the future we will evolve our generative approach to CVE adopting the Object Management Group technology, i.e. formalizing the concept map as Meta Object Facility meta-model and exploiting model-to-model and model-to-code (e.g. Acceleo) techniques to configure virtual environments instances to support cultural exploration.

Acknowledgment. The work is partially funded by the Italian Ministry of Education, University and Research (MIUR) under the PON4a2 DICET-INMOTO (LivingLab on Culture and Technology - Information and Mobility for Tourism) national research project.

\section{References}

1. Cucchiara, R., Grana, C., Borghesani, D., Agosti, M., Bagdanov, A.D.: Multimedia for Cultural Heritage: Key Issues. In: Grana, C., Cucchiara, R. (eds.) MM4CH 2011. CCIS, vol. 247, pp. 206-216. Springer, Heidelberg (2012)

2. Di Blas, N., Bucciero, A., Mainetti, L., Paolini, P.: Multi-User Virtual Environments for Learning: Experience and Technology Design. IEEE Transactions on Learning Technologies 4(5), 349-365 (2012)

3. Kurillo, G., Forte, M., Bajcsy, R.: Teleimmersive 3D Collaborative Environment for Cyberarchaeology. In: Computer Vision and Pattern Recognition Workshops (CVPRW), pp. 23-28. IEEE Press, New York (2010)

4. Vlahakis, V., et al.: Archeoguide: first results of an augmented reality, mobile computing system in cultural heritage sites. In: 2001 Conference on Virtual Reality, Archeology, and Cultural Heritage, pp. 131-140. ACM, New York (2001) 
5. Corlàita, D., et al.: Exciting understanding in Pompeii through on-site parallel interaction with dual time virtual models. In: 2001 Conference on Virtual Reality, Archeology, and Cultural Heritage, pp. 83-90. ACM, New York (2001)

6. Hall, T., et al.: The visitor as virtual archaeologist: explorations in mixed reality technology to enhance educational and social interaction in the museum. In: 2001 Conference on Virtual Reality, Archeology, and Cultural Heritage, pp. 91-96. ACM, New York (2001)

7. Guerrero, L.A., Collazos, C.A., Pino, J.A., Ochoa, S.F., Aguilera, F.: Designing collaborative virtual environments based on real spaces to promote community interaction. In: First Latin American Web Congress, pp. 58-65 (2003)

8. Rossi, D., Turrini, E.: Using a process modeling language for the design and implementation of process-driven applications. In: ICSEA 2007, Cap Esterel, France, pp. 55-55 (2007)

9. Vanacken, D., De Boeck, J., Raymaekers, C., Coninx, K.: NiMMiT: A notation for modeling multimodal interaction techniques. In: International Conference GRAPP 2006, Setúbal, Portugal, pp. 224-231 (2006)

10. Di Blas, N., Poggi, C., Torrebruno, A.: Collaboration and Playful Competition in a 3D Educational Virtual World: The Learning@Europe Experience. In: World Conference on Educational Multimedia, Hypermedia and Telecommunications, pp. 1191-1198. AACE, Chesapeake (2006)

11. Barchetti, U., Barbieri, T., Bucciero, A., Mainetti, L., Santo Sabato, S.: WebTalk04: a Framework to Support 3D Collaborative e-Learning. In: WBE 2006 - 5th IASTED International Conference on Web-based Education, Puerto Vallarta (Mexico), pp. 13-18 (2006) Barchetti, U., Bucciero, A., De Benedittis, T.A., Macchia, F., Mainetti, L., Tamborino, A.: MoWeT: A Configurable Framework to Support Ubiquitous Location-Aware Applications. In: The Sixth International Conference on Ubiquitous Computing and Intelligence, Brisbane, Australia, pp. 75-82 (2009) 